\title{
Elliptical ASIFT Agglomeration in Class Prototype for Logo Detection
}

\section{Raluca Boia}

rboia@imag.pub.ro;

Corneliu Florea

corneliu.florea@upb.ro;

Laura Florea

laura.florea@upb.ro;

\author{
Image Processing and Analysis Laboratory \\ University "Politehnica" of Bucharest \\ Bucharest, Romania
}

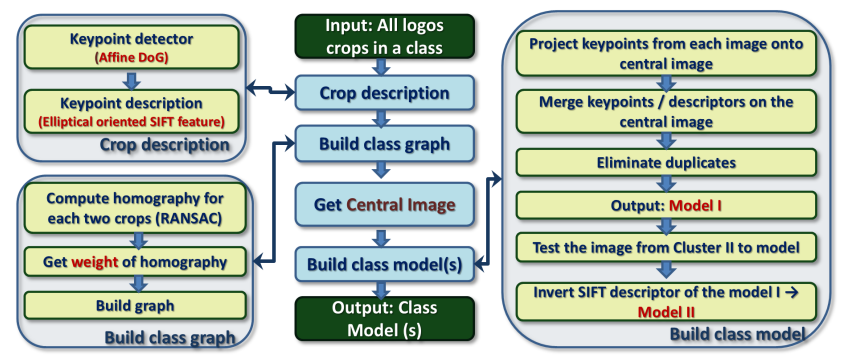

Figure 1: The schematic of the proposed system while describing the classes. With red are specific choices leading to greater performance.

Logo (graphic entity that contains colors, shapes, textures and identifies organizations, goods, etc.) localization and recognition is a subproblem of object detection and recognition and a challenging pattern recognition task. Applications are in the automotive industry, sports transmissions, legal or feedback for advertising.

Logos in natural images are approached within retrieval systems [1] [6], [4], etc. or by integrated detection (localization and recognition) [2], [7], etc. Our contribution falls in the second category and consists in: (1) a new class prototyping method based on a central image extracted by analyzing the homographies graph and re-projecting the relevant keypoints on that image (2) a logo detection system that exhibits great performance. The main conceptual difference to previous systems is that they manually branched their process to deal with corner-cases, while we perform the branching automatically, proposing a compact and self-adjusting system.

Class Description. In the training phase (detailed in Fig. 1) we construct models (prototypes) to describe classes. In the testing phase (Fig. 2) the query image is compared with class prototypes and if they are enough similar we count a detection.

Feature extraction. The logo images are described by the Affine Difference of Gausssians (ADoG) [5] followed by the description with oriented SIFT elliptical local features. ADoG provides more keypoints on logos than other choices, while elliptical features are able to provide correctly the orientation even for circular logos.

Class Graph. All the logo crops from the same class are grouped in a weighted graph: the nodes are the logos, while an edge is created if a homography is found between that pair of logos-nodes. The edge has an weight equal to the inverse of the number of keypoints pairs matched.

The homography between two logos is found with the direct linear transformation (DLT) and 4 keypoint pairs are needed for this determination. The matching between the reference logo and the subject logo is found with RANSAC. Yet, to provide the best match, one needs to iterate more subsets than usual [3].

Next, the quality of the homographic fit is evaluated using an error map built as the Hellinger distance between the reference logo and the back-projected logo described with Dense SIFT.

Given the class graph, the central image is the node with the most connections.

Class Model. The class model is built by agglomerating onto the central image the suitable keypoints and their SIFT description. This information is taken from all the logo images from the main cluster of the class graph, by projecting them on the plane of the central image.

Keypoints in images directly connected to the central image are backprojected (by inverting the matching homography) on the central one. The equivalent homography between images that are not directly connected to the central image is determined by composing the homographies placed on the path between that image and the central one. The chosen path is the one that ensures minimum cumulative weight. The corresponding SIFT

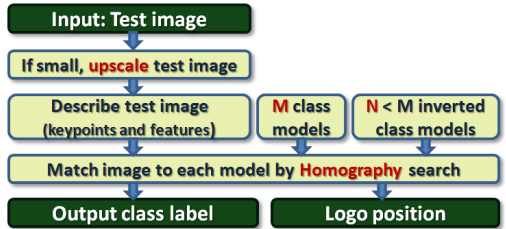

Figure 2: The schematic of the system used to locate and classify a logo in a testing image.

descriptors are merged on the central image, as they are not affected by the projection into another plane.

Color Inverted Logos: Secondary Prototype. To deal with classes containing inverted colors, a secondary model is build. If the graph contains a secondary cluster not connected to the first, the existence of color inverted case is detected and a secondary model is built by inverting the SIFT feature orientation of the main prototype.

Results. In the testing phase, we locate logos and classify them. Given at least one model for each class, we use RANSAC to match the keypoints from the query image against all the class models as shown in figure 2. To decide whether the match is correct, the average of the error map based on Dense SIFT is computed for each matching result. If, after being confronted to all the class models, no score is large enough, then the test image will be classified as "no-logo". To be able to detect small size logos, we upscale the original image 4 times.

Evaluation Procedure. Given a query image, we count a true detection if the found logo is indeed in the image and if the intersection-overunion is above $50 \%$.

The classification rates on the tested databases are: on FlickrLogos32: $93.33 \%$ (9.27\% improvement to state of the art), BelgaLogos: $78.09 \%$ (11.76\% improvement) and FlickrLogos-27: $72.8 \%$ (1.2\% improvement). The method does not produces any false positives on the testing datasets, but only miss detections.

The failures are associated with logo classes with few keypoints, small logos or with extreme warping or blurring. Another situation of poor performance is for logos that are rather different from the training set.

The algorithm is also able to detect multiple logos in an image by testing the query image against all class models and retaining all homography matches that produced an average of the error map smaller than chosen threshold. Creating additional virtual classes for cases which present high variation in color (including both original and negative version) permits simultaneous detection of both cases, even in the same image.

[1] A. Bagdanov, L. Ballan, M. Bertini, and A. Del Bimbo. Trademark matching and retrieval in sports video databases. In ACM MIR, 2007.

[2] R. Boia and C. Florea. Homographic class template for logo localization and recognition. In Proc. of IbPRIA, pages 487-495, 2015.

[3] M. Brown and D. Lowe. Automatic panoramic image stitching using invariant features. IJCV, 74(1):59-73, 2007.

[4] J. Krapac, F. Perronnin, T. Furon, and H. Jegou. Instance classification with prototype selection. In ACM ICMR, pages 431 - 4, 2014.

[5] J.M. Morel and G.Yu. ASIFT: A new framework for fully affine invariant image comparison. SIIMS, 2(2):438-469, 2009.

[6] C. Ries, F. Richter, S. Romberg, and R. Lienhart. Automatic object annotation from weakly labeled data with latent structured SVM. In CBMI, pages 1-4, 2014.

[7] H. Sahbi, L. Ballan, G. Serra, and A. Del Bimbo. Context-dependent logo matching and recognition. IEEE TIP, 22:1018-1031, 2013. 